\title{
LUPUS SCIENCE\& MEDICINE \\ TAC-TIC use of tacrolimus-based regimens in lupus nephritis
}

\author{
Tineke Kraaij, ${ }^{1,2}$ Obbo W Bredewold, ${ }^{1,2}$ Stella Trompet, ${ }^{3,4}$ Tom W J Huizinga, ${ }^{2,5}$ \\ Ton J Rabelink, ${ }^{1,2}$ Anton J M de Craen, ${ }^{3, \dagger}$ Y K Onno Teng ${ }^{1,2}$
}

To cite: Kraaii T, Bredewold OW, Trompet S, et al. TAC-TIC use of tacrolimus-based regimens in lupus nephritis. Lupus Science \& Medicine 2016;3: e000169. doi:10.1136/lupus2016-000169

- Additional material is available. To view please visit the journal online (http://dx. doi.org/10.1136/lupus-2016000169).

Dr Craen died on January 17th, 2016.

Received 10 June 2016 Revised 1 December 2016 Accepted 3 December 2016

\section{ABSTRACT}

Current guidelines do not mention tacrolimus (TAC) as a treatment option and no consensus has been reported on the role of TAC in lupus nephritis (LN). The present study aimed to guide clinical judgement on the use of TAC in patients with LN. A meta-analysis was performed for clinical studies investigating TAC regimens in $\mathrm{LN}$ on the basis of treatment target (induction or maintenance), concomitant immunosuppression and quality of the data. 23 clinical studies performed in patients with $\mathrm{LN}$ were identified: 6 case series, 9 cohort studies, 2 case-control studies and 6 randomised controlled trials (RCTs). Of the 6 RCTs, 5 RCTs investigated TAC regimens as induction treatment and 1 RCT as maintenance treatment. Five RCTs investigated TAC in combination with steroids and 2 TAC with mycophenolate plus steroids. All RCTs were performed in patients of Asian ethnicity. In a meta-analysis, TAC regimens achieved a significantly higher total response (relative risk (RR) $1.23,95 \% \mathrm{Cl}$ 1.12 to $1.34, p<0.05$ ) and significantly higher complete response (RR $1.48,95 \% \mathrm{Cl} 1.23$ to $1.77, \mathrm{p}<0.05$ ). The positive outcome was predominantly defined by the largest RCT investigating TAC with mycophenolate plus steroids. Regarding safety, the occurrence of leucopoenia was significantly lower, while the occurrence of increased creatine was higher. Clinical studies on TAC regimens for $L N$ are limited to patients of Asian ethnicity and hampered by significant heterogeneity. The positive results on clinical efficacy of TAC as induction treatment in $\mathrm{LN}$ cannot be extrapolated beyond Asian patients with LN. Therefore, further confirmation in multiethnic, randomised trials is mandatory. Until then, TAC can be considered in selected patients with LN.

\section{INTRODUCTION}

Lupus nephritis (LN) occurs in up to $60 \%^{1}$ of all patients with systemic lupus erythematosus (SLE) and is associated with increased mortality rates. ${ }^{2}$ Current guidelines on the treatment for $\mathrm{LN}$ recommend corticosteroids in combination with cyclophosphamide or mofetil mycophenolate (MMF) as induction treatment and azathioprine or MMF as maintenance treatment. ${ }^{3}{ }^{4}$ Nevertheless, there is a persistent need for new therapeutic options since the cumulative renal flare rate is $50 \%$ within 10 years upon the first-choice conventional treatments. ${ }^{5}$ For these refractory patients, guidelines are less specific in their recommendations: Rituximab is most often recommended to be considered despite the negative results in randomised trials. ${ }^{6}{ }^{7}$ Interestingly, no consensus was reached on the role of calcineurin inhibitors $(\mathrm{CNIs})^{3}{ }^{4}$ despite two recently published, large randomised controlled trials (RCTs) showing a positive signal on the efficacy of a tacrolimus (TAC)-based treatment in LN. ${ }^{8}{ }^{9}$ Moreover, an attractive aspect of TAC is that it also can be given during pregnancy, ${ }^{10}{ }^{11}$ which is a frequent dilemma in young women with SLE. Also, TAC is a readily available agent and commonly used in kidney transplantation. Taken together, systematically analysing the potential role of TAC as treatment for LN is necessary.

TAC is a macrolide CNI frequently used in solid organ transplantation to prevent rejection. ${ }^{12}$ Calcineurin inhibition by TAC prevents dephosphorylation of the nuclear factor of activated $T$ cells and thereby reduces activity of genes coding interelukin 2 and related cytokines, ${ }^{13}$ leading to inhibition of T cell activation. Besides its immunosuppressive effect TAC, as well as its calcineurin-inhibiting predecessor ciclosporine, are both known for their antiproteinuric effects in treating a variety of renal pathologies. ${ }^{14}$ In an SLE mouse model,${ }^{15}$ treatment with TAC in animals with spontaneous LN shows inhibition of the progression of glomerular hypercellularity, crescent formation, proteinuria development and suppression of serum anti-dsDNA antibody elevation. Thus, from an immunological point of view, TAC might have potential as treatment for LN.

The present study aimed to guide clinical judgement on the use of TAC in patients with LN. Therefore, we systematically reviewed all the published clinical studies that investigated a TAC regimen in $\mathrm{LN}$ and performed a meta-analysis on the efficacy of TAC regimens and assessed available safety parameters. 


\section{METHODS}

Literature search strategy and data analysis

Pubmed, Embase, Web of Science and Cochrane databases were searched for all human studies on treatment of LN with TAC. The following search terms were used: ((('Tacrolimus'[Mesh] OR 'tacrolimus' [tw] OR tacrolimus*[tw] OR 'Prograf' [tw] OR 'Prograft' [tw] OR 'FR-900506' $[\mathrm{tw}]$ OR 'FR 900506'[tw] OR 'FR900506' [tw] OR 'FK-506' [tw] OR 'FK 506' [tw] OR 'FK506' [tw] OR 'WM0H WNM'[all fields]) AND ('Nephritis' [Mesh] OR 'nephritis' [tw] OR nephrit*[tw] OR 'Glomerulonephritis' [tw] OR 'Anti-Glomerular Basement Membrane Disease' $[\mathrm{tw}] \quad$ OR 'Glomerulosclerosis' [tw] OR 'Balkan Nephropathy'[tw] OR 'Pyelonephritis' [tw] OR 'Pyelitis'[tw] OR 'Pyelocystitis' $[\mathrm{tw}]$ ) AND ('Lupus Erythematosus, Systemic'[Mesh] OR 'Systemic Lupus Erythematosus' [tw] OR 'SLE' [tw] OR 'lupus' $[\mathrm{tw}])$ ) OR (('Tacrolimus'[Mesh] OR 'tacrolimus' [tw] OR tacrolimus*[tw] OR 'Prograf' $[\mathrm{tw}]$ OR 'Prograft' $[\mathrm{tw}]$ OR 'FR-900506'[tw] OR 'FR 900506'[tw] OR 'FR900506' [tw] OR 'FK-506' [tw] OR 'FK 506'[tw] OR 'FK506' [tw] OR 'WM0H WNM'[all fields]) AND ('Lupus Nephritis' [Mesh] OR 'Lupus Nephritis' [tw] OR 'Lupus Glomerulonephritis' $[\mathrm{tw}]))$ ) AND ('Clinical Trial'[publication type] OR random*[tw] OR 'trial' [tw] OR 'RCT'[tw] OR placebo*[tw] OR 'double blind' $[t w])$. According to the Preferred Reporting Items for Systematic Reviews and Meta-Analyses (PRISMA) criteria, ${ }^{16}$ titles and abstracts of search results were evaluated for suitability based on the following criteria: (1) published as a clinical trial in human subjects; (2) included patients had an established diagnosis of SLE in accordance with the American College of Rheumatology revised criteria; (3) the presence of $\mathrm{LN}$ and persistent clinical findings such as elevated serum creatine, proteinuria $>0.5 \mathrm{~g}$ or active urine sediment; (4) for controlled studies: well defined renal complete, partial and non-response criteria. The studies were judged and selected independently by two investigators (TK and YKOT). Consensus was achieved on studies that were selected by only one of two investigators.

All studies were labelled according to their design, that is: 'case series' when 10 or less patients were reported, 'uncontrolled cohort' when more than 10 patients were studied, 'case-control study' (CCS) or 'randomised controlled trial' (RCT). Study characteristics were summarised by descriptive statistics and ordered on the basis of type and goal of TAC treatment leading to four categories: (1) studies applying a TAC regimen as induction treatment for new $\mathrm{LN}$ or flare of $\mathrm{LN}$; (2) studies applying a TAC regimen as maintenance treatment for patients with $\mathrm{LN}$ who had received any given induction treatment; (3) studies applying a TAC regimen applied as induction treatment and followed by (lower dosages of) TAC as maintenance treatment; (4) studies switching conventional treatment to a TAC regimen during the maintenance phase. The quality of randomised controlled trials was assessed with the Delphi list. $^{17}$

From all controlled studies relevant variables were extracted, that is, baseline characteristics, trial design characteristics, TAC regimen characteristics, renal responses, dropouts and adverse events. With respect to renal response criteria, the definitions for complete, partial and no response were adapted from the individual studies.

\section{Statistical analysis}

Descriptive statistics were used to summarise baseline, trial and TAC regimen characteristics. Data from five RCTs were used in a meta-analysis, to compare renal response and adverse events between TAC-based regimens and control therapy. The meta-analysis was performed with Stata, V.10 (Statacorp, Texas, USA). The relative risk (RR) and 95\% CI for each outcome was calculated for each study using the Mantel-Haenszel fixedeffects model. Heterogeneity was determined by the $\chi^{2}$ and $\mathrm{I}^{2}$ tests. An outcome of $\mathrm{p}<0.05$ was considered a significant difference.

\section{RESULTS}

\section{Summary of the literature search}

Our search strategy resulted in 239 articles of which 23 relevant clinical studies were selected based upon the predefined quality criteria (figure 1). As depicted in table 1 , we found that the majority of clinical studies consisted of uncontrolled case series $(26 \%)$ and uncontrolled cohort studies (39\%). Controlled studies encompassed 2 (9\%) CCS and $6(26 \%)$ RCTs. From all selected studies, $87 \%$ were exclusively performed in Asian LN populations, leaving 3 (13\%) uncontrolled

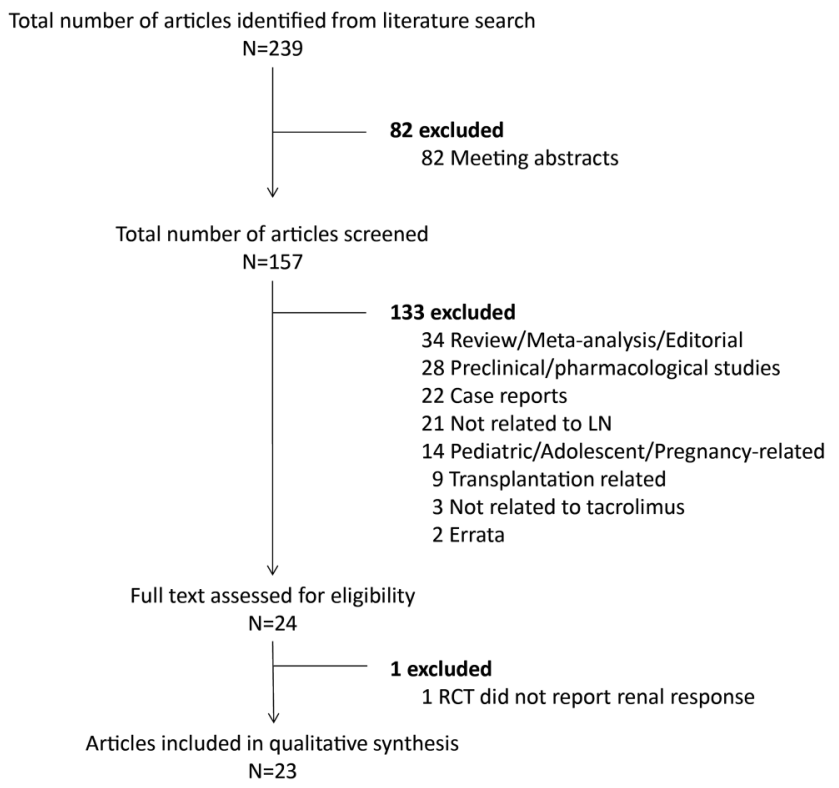

Figure 1 Flow chart of the literature search. LN, lupus nephritis; RCT, randomised controlled trial. 
Table 1 Summary of study characteristics

\begin{tabular}{lc}
\hline Study characteristics (N=23) & (\%) \\
\hline Design & \\
Case series $(\mathrm{N} \leq 10)$ & $6(26)$ \\
Uncontrolled cohort (N>10) & $9(39)$ \\
Case-control study & $2(9)$ \\
Randomised controlled trial & $6(26)$ \\
Subjects & \\
Asian alone & $20(87)$ \\
Non-Asian & $3(13)$ \\
Regimen & \\
Tacrolimus+steroids & $15(65)$ \\
Tacrolimus+steroids+mycophenolate & $6(26)$ \\
Tacrolimus+steroids+mizoribine & $2(9)$ \\
Tacrolimus used as & \\
Induction therapy & $13(57)$ \\
Maintenance therapy & $5(22)$ \\
Induction and maintenance therapy & $3(13)$ \\
Therapy switch* & $2(9)$ \\
*Study was designed to switch patients from conventional \\
treatment to a tacrolimus-based regimen.
\end{tabular}

studies in non-Asian patients. The most frequently studied TAC regimen combined TAC with steroids $(65 \%)$, also termed 'duo therapy'. Six (26\%) studies combined TAC with steroids plus MMF, also termed 'triple therapy'. A majority of $13(57 \%)$ studies investigated TAC as induction treatment, $5(22 \%)$ as maintenance treatment, $3(13 \%)$ used TAC as induction and subsequent maintenance treatment and $2(9 \%)$ studies investigated a switch of conventional maintenance to a TAC maintenance regimen.

Table 2 summarises the controlled studies grouped by treatment goal and on the basis of their treatment regimen. This overview illustrates the heterogeneity of the published studies.

To better understand the studied TAC regimens in the controlled studies, the quality score (only applicable in RCT), study designs and TAC dosing were summarised in table 3. Overall, the quality of studies was poor to average (median score 4, range 3-5) as measured by the standardised Delphi scoring for RCTs. Importantly, one shared characteristic was that all studies investigating induction treatment with TAC regimens defined their renal response end point at 6 months. The definition of renal response, however, was different for each study (see online supplementary table S2). With respect to dosing, we could not find any coherence between any of the studies nor within studies investigating duo therapy or triple therapy TAC regimens. Seven $(88 \%)$ studies measured TAC trough levels to guide their dosing, however target trough levels varied per study (table 3).

\section{Patient characteristics}

Overall, 693 patients were included in the meta-analysis on renal response and most frequently reported adverse events. Patient characteristics were summarised in table 4 .
In short, $90 \%$ of the subjects were female. The mean age was 32 years and $100 \%$ were of Asian ethnicity. Histopathologically, $84 \%$ had an LN class III/IV $\pm \mathrm{V}$ and $16 \%$ LN class V.

Of note, for non-Asian subjects we did not find any controlled trials. Two case series ${ }^{24} 25$ and one uncontrolled cohort study ${ }^{26}$ with a total of 32 patients using TAC regimens have been published and none met the selection criteria.

\section{Meta-analysis of renal responses upon induction treatment with TAC-based regimens}

The results of the meta-analysis are shown in figure 2. Five RCTs investigated TAC regimens in the induction treatment phase and were used for data extraction. Again, all studies reported renal response rate as a primary end point at 6 months. Three RCTs used intravenous cyclophosphamide in the control arm, ${ }^{9} 1820$ one study mycophenolate ${ }^{8}$ and one study ${ }^{19}$ contained two control arms using either mycophenolate or cyclophosphamide. TAC-based induction treatment led to a significantly higher total renal response (RR $1.23,95 \%$ CI 1.12 to $1.34, \mathrm{p}<0.05)$ with significantly higher complete renal response (RR 1.48, 95\% CI 1.23 to $1.77, \mathrm{p}<0.05$ ) and equivalent partial renal response (RR $0.98,95 \%$ CI 0.79 to $1.21, \mathrm{p}=$ not significant (NS)). The RR for total, complete and partial response was also assessed for studies using duo therapy and triple therapy separately. In RCTs using duo therapy, TAC-based induction treatment led to equivalent total renal response (RR 1.06, 95\% CI 0.94 to $1.19, \mathrm{p}=\mathrm{NS}$ ) with equivalent complete renal responders (RR $1.15,95 \%$ CI 0.92 to $1.44, \mathrm{p}=\mathrm{NS}$ ) as well as partial responders (RR $0.91,95 \%$ CI 0.62 to $1.34, \mathrm{p}=\mathrm{NS}$ ). For RCTs using triple therapy, TAC-based induction treatment led to a significantly higher total renal response (RR $1.37,95 \%$ CI 1.21 to $1.56, \mathrm{p}<0.05$ ), with more complete responders (RR 1.94, 95\% CI 1.45 to $2.61, \mathrm{p}<0.05$ ), and equivalent partial responders (RR 1.01, 95\% CI 0.78 to $1.31, \mathrm{p}=\mathrm{NS})$.

\section{Renal responses upon maintenance treatment with TAC} and steroids

Only one study ${ }^{21}$ met our quality criteria to evaluate the effect of maintenance treatment with a TAC regimen. This study reported an equivalent response of $100 \%$ vs 95\% to TAC versus control treatment after 6 months: $56 \%$ achieved a complete remission (19 out of 34$)$ and $44 \%$ achieved a partial remission (15 out of 34 ). No flares were observed during this period. In the control group, where patients received azathioprine, 64\% achieved complete remission (23 out of 36 ) and $31 \%$ a partial remission (11 out of 36). Two flares were observed in the control arm.

\section{Meta-analysis of adverse events upon induction treatment} with TAC and steroids

From the five RCTs investigating TAC regimens in the induction phase, the most frequently reported adverse 
Table 2 Number of studies stratified by treatment intention

\begin{tabular}{|c|c|c|c|}
\hline & $\begin{array}{l}\text { Tacrolimus } \\
\text { +steroids } \\
\end{array}$ & $\begin{array}{l}\text { Tacrolimus+steroids } \\
\text { +mycophenolate }\end{array}$ & $\begin{array}{l}\text { Tacrolimus+steroids } \\
\text { +mizoribine }\end{array}$ \\
\hline Induction therapy & $\begin{array}{l}7 \\
3 R C T s\end{array}$ & $\begin{array}{l}4 \\
2 R C T s\end{array}$ & 2 \\
\hline Maintenance therapy & 4 & 2 & 0 \\
\hline $\begin{array}{l}\text { Induction and maintenance } \\
\text { therapy }\end{array}$ & $2 \operatorname{ccs}$ & 0 & 0 \\
\hline Therapy switch & 2 & 0 & 0 \\
\hline
\end{tabular}

Table 3 Overview of the studies fulfilling the predefined selection criteria for analysis of tacrolimus-based regimens in patients with lupus nephritis

\begin{tabular}{|c|c|c|c|c|c|}
\hline & $\begin{array}{l}\text { Type of } \\
\text { study }\end{array}$ & $\begin{array}{l}\text { Quality } \\
\text { score }(0-9)^{\star}\end{array}$ & $\begin{array}{l}\text { No. of } \\
\text { patients }\end{array}$ & $\begin{array}{l}\text { Time to } \\
\text { end point }\end{array}$ & Treatment regimen \\
\hline \multicolumn{6}{|c|}{ Induction with duo therapy } \\
\hline Chen et $a l^{18}$ & $\mathrm{RCT}$ & 5 & 81 & 6 months & $\begin{array}{l}\text { TAC: blood concentration of } 5-10 \mathrm{ng} / \mathrm{mL} \\
\text { Pred: initial dose } 1 \mathrm{mg} / \mathrm{kg} / \text { day (max. } 60 \mathrm{mg} / \text { day) tapered } \\
\text { until } 10 \mathrm{mg} / \text { day }\end{array}$ \\
\hline Li et $a l^{19}$ & $\mathrm{RCT}$ & 4 & 60 & 6 months & $\begin{array}{l}\text { TAC: blood concentration of } 6-8 \mathrm{ng} / \mathrm{mL} \\
\text { Pred: initial dose } 1 \mathrm{mg} / \mathrm{kg} / \text { day (max. } 60 \mathrm{mg} / \mathrm{day} \text { ), tapered } \\
\text { until } 10 \mathrm{mg} / \text { day }\end{array}$ \\
\hline Mok et $a \beta^{\beta}$ & $\mathrm{RCT}$ & 4 & 150 & 6 months & $\begin{array}{l}\text { TAC: } 0.1 \mathrm{mg} / \mathrm{kg} / \mathrm{day} \text { reduced to } 0.06 \mathrm{mg} / \mathrm{kg} / \mathrm{day} \text { at } \\
3 \mathrm{months} \text { if clinical response is satisfactory } \\
\text { Pred: initial dose } 0.6 \mathrm{mg} / \mathrm{kg} / \text { day for } 6 \text { weeks, tapered until } \\
<10 \mathrm{mg} / \text { day }\end{array}$ \\
\hline \multicolumn{6}{|c|}{ Induction with triple therapy } \\
\hline Bao et $a^{R o}$ & $\mathrm{RCT}$ & 5 & 40 & 6 months & $\begin{array}{l}\text { TAC: blood concentration of } 5-7 \mathrm{ng} / \mathrm{mL} \\
\text { MMF: } 1.0 \mathrm{~g} / \text { day, (AUC) } 20-45 \mathrm{mg} \text { hour } / \mathrm{L} \\
\text { Intravenous methylprednisone: } 0.5 \mathrm{~g} / \text { day for } 3 \text { days } \\
\text { Pred: pred } 0.6-0.8 \mathrm{mg} / \mathrm{kg} / \text { day for } 4 \text { weeks, tapered until } \\
\text { maintenance dose } 10 \mathrm{mg} / \text { day }\end{array}$ \\
\hline Liu et aP & $\mathrm{RCT}$ & 5 & 362 & 6 months & $\begin{array}{l}\text { TAC: adjusted according to blood concentration } \\
\text { measured throughout the study } \\
\text { MMF: according to AUC measured throughout the study } \\
\text { Pred: similar between treatment groups, gradually } \\
\text { tapered }\end{array}$ \\
\hline \multicolumn{6}{|c|}{ Maintenance with duo therapy } \\
\hline Chen et $a^{P^{1}}$ & $\mathrm{RCT}$ & 4 & 70 & 6 months & $\begin{array}{l}\text { TAC: blood concentrations of } 4-6 \mathrm{ng} / \mathrm{mL} \\
\text { Pred: } 10 \mathrm{mg} / \text { day }\end{array}$ \\
\hline \multicolumn{6}{|c|}{ Induction and maintenance with duo therapy } \\
\hline Yap et $a^{22}$ & CCS & NA & 16 & 24 months & $\begin{array}{l}\text { TAC: blood concentration of } 6-8 \mathrm{ng} / \mathrm{mL} \text { in the first } \\
6 \mathrm{months} ; 5-5.9 \mathrm{ng} / \mathrm{mL} \text { in the next } 6 \text { months; } 3.0-4.9 \\
\mathrm{ng} / \mathrm{mL} \text { in the last year } \\
\text { Pred: } 0.8 \mathrm{mg} / \mathrm{kg} / \text { day (maximum } 50 \mathrm{mg} / \text { day), tapered to } \\
7.5 \mathrm{mg} / \text { day until end of study (in patients }<50 \mathrm{~kg} \text { reduced } \\
\text { to } 5 \mathrm{mg} / \text { day) }\end{array}$ \\
\hline Wang et $a^{23}$ & CCS & NA & 40 & 12 months & $\begin{array}{l}\text { TAC: blood concentration of } 6-8 \mathrm{ng} / \mathrm{mL} \text { during induction, } \\
4-6 \mathrm{ng} / \mathrm{mL} \text { during maintenance } \\
\text { Pred: } 0.8 \mathrm{mg} / \mathrm{kg} / \text { day (maximum } 50 \mathrm{mg} / \text { day), tapered until } \\
10-15 \mathrm{mg} / \text { day during maintenance }\end{array}$ \\
\hline
\end{tabular}

${ }^{*}$ Quality assessed with the Delphi score.

AUC, area under curve; CCS, case-control study; MMF, mycophenolate mofetil; NA, not applicable; pred, prednisone; RCT, randomised controlled trial; TAC, tacrolimus. 
Table 4 Baseline characteristics of patients with LN from the selected RCTs that are used in the meta-analysis for renal response and adverse events

\begin{tabular}{llll} 
& & \multicolumn{2}{l}{ Induction therapy } \\
\cline { 3 - 4 } & All & $\begin{array}{l}\text { Duo } \\
\text { therapy }\end{array}$ & $\begin{array}{l}\text { Triple } \\
\text { therapy }\end{array}$ \\
\hline $\mathrm{N}^{*}$ & 693 & 291 & 402 \\
Age $^{*}$ & 32 & 33 & 32 \\
Female (\%) & 90 & 89 & 90 \\
Disease duration (years) & 1.6 & 3.2 & 0.5 \\
Asian ethnicity (\%) & 100 & 100 & 100 \\
LN class (\%) & & & \\
$\quad$ I/II & & & \\
$\quad$ III/IV $\pm V$ & 84 & 85 & 83 \\
$\quad$ V & 16 & 15 & 17 \\
*Data are expressed as the mean. & & \\
LN, lupus nephritis. & & &
\end{tabular}

events were included for meta-analysis (figure 3). Leucopoenia was significantly less reported in the TAC-based treatment group (RR 0.21 , 95\% CI 0.08 to $0.54, \mathrm{p}<0.05)$. A rise of serum creatine was higher in the TAC-based treatment group (RR 6.29, 95\% CI 1.79 to 22.09, $\mathrm{p}<0.05)$. Infectious complications were comparable between the TAC-based treatment group and control group (RR $0.91,95 \%$ CI 0.69 to $1.19, \mathrm{p}=\mathrm{NS}$ ). Although severe infections (RR $0.90,95 \%$ CI 0.48 to $1.69, \mathrm{p}=\mathrm{NS}$ ) and hyperglycaemia (RR $1.40,95 \%$ CI 0.78 to $2.52, \mathrm{p}=\mathrm{NS}$ ) were more often reported in the TAC-based treatment group, these results did not reach statistical significance. Relative risks for the most reported adverse events were also compared between duo therapy and triple therapy separately. Overall, results between studies using duo therapy or triple therapy did not differ. Importantly, the TAC-based treatment in the RCTs using duo therapy showed a lower, non-significant rate for severe infection (RR 0.42, 95\% CI 0.17 to $1.03, \mathrm{p}=\mathrm{NS}$ ), whereas a trend to a higher rate of severe infections was seen with triple therapy (RR $2.83,95 \%$ CI 0.92 to $8.72, \mathrm{p}=\mathrm{NS}$ ).

\section{DISCUSSION}

The present study was performed to better guide clinical judgement on the use of TAC in patients with LN. Selecting only the highest quality studies for meta-analysing the clinical efficacy of TAC-based regimen, we demonstrated that the currently available studies are predominantly non-randomised, uncontrolled studies. Our systematic meta-analysis of randomised trials comparing TAC-based regimens with conventional treatment demonstrated superior efficacy in Asian patients with LN, mainly determined by studies evaluating triple therapy. ${ }^{920}$ Safety profiles of TAC-based regimens were comparable to conventional treatment. These results cannot be extrapolated to the general LN population. Therefore, taken all together, current evidence supports the use of TAC-based regimens in a selected group of patients with LN of Asian ethnicity with a preference for using triple therapy (TAC, MMF and steroids) as induction treatment. The latter said, long-term safety of TAC-based regimens is not established.

The goal of this study was to translate published study results on TAC in LN to current clinical practice. Based on our study and previous meta-analyses ${ }^{27} 28$ there is level $1 \mathrm{~A}$ evidence ${ }^{29}$ to support the clinical efficacy of TAC in the subgroup of Asian patients with LN. However, our study illustrated that a 'grade A' recommendation for TAC is hampered by the heterogeneity of TAC-based regimens studied in this subgroup of patients with LN. In this view it is important to note that the positive result of our meta-analysis was predominantly determined by the study of Liu et at that investigated a TAC-based regimen using 'triple' therapy combining steroids, mycophenolate and TAC. Altogether, it is selfevident that a randomised, multiethnic study is mandatory to further expand our knowledge and evidence of TAC treatment in LN.

To further guide clinicians in the use of TAC, it is reasonable to extrapolate the level 1A evidence (see online supplementary table S1) described above to the subgroup of refractory patients with LN. Generally, refractory LN is defined as a failure on two conventional treatments (being either mycophenolate or cyclophosphamide). ${ }^{3} 430$ Several treatment suggestions are made in $\mathrm{LN}$ treatment guidelines for refractory $\mathrm{LN}$ such as rituximab, CNIs, intravenous immunoglobulins, plasmapheresis and tumor necrosis factor (TNF) blockade. ${ }^{31}$ Thus, with respect to TAC, a grade $\mathrm{B}$ positive recommendation can be formulated. Our data at the least suggest that the use of TAC is not inferior to conventional treatment. Moreover, we and others ${ }^{27} 2832$ showed that the safety profile of TAC is very good in LN. Therefore, we would recommend TAC to be considered as a treatment option in patients with refractory LN.

TAC is a safe drug during pregnancy and its continuation is commonly recommended in the setting of pregnant patients who have received solid organ transplantation. ${ }^{10}$ 33-35 From this perspective, the level 1A evidence on the efficacy of TAC in Asian patients with LN should also be considered for extrapolation to this special subgroup of patients with LN. Although TAC is non-teratogenic, there is an increased risk of gestational diabetes and hypertension. ${ }^{36}$ Currently, there are no controlled studies available investigating TAC for LN in pregnant patients. In a case series on nine patients with LN, ${ }^{33}$ TAC was successfully used to maintain remission in three patients and to treat a lupus flare in six patients. All pregnancies resulted in live births with birth weights according to gestational age and no congenital abnormalities. At present, azathioprine is considered the first choice of treatment in pregnant patients with LN. ${ }^{37}$ However, in those patients with LN who are azathioprine-resistant or azathioprine-intolerant, TAC can be considered as a treatment option. 

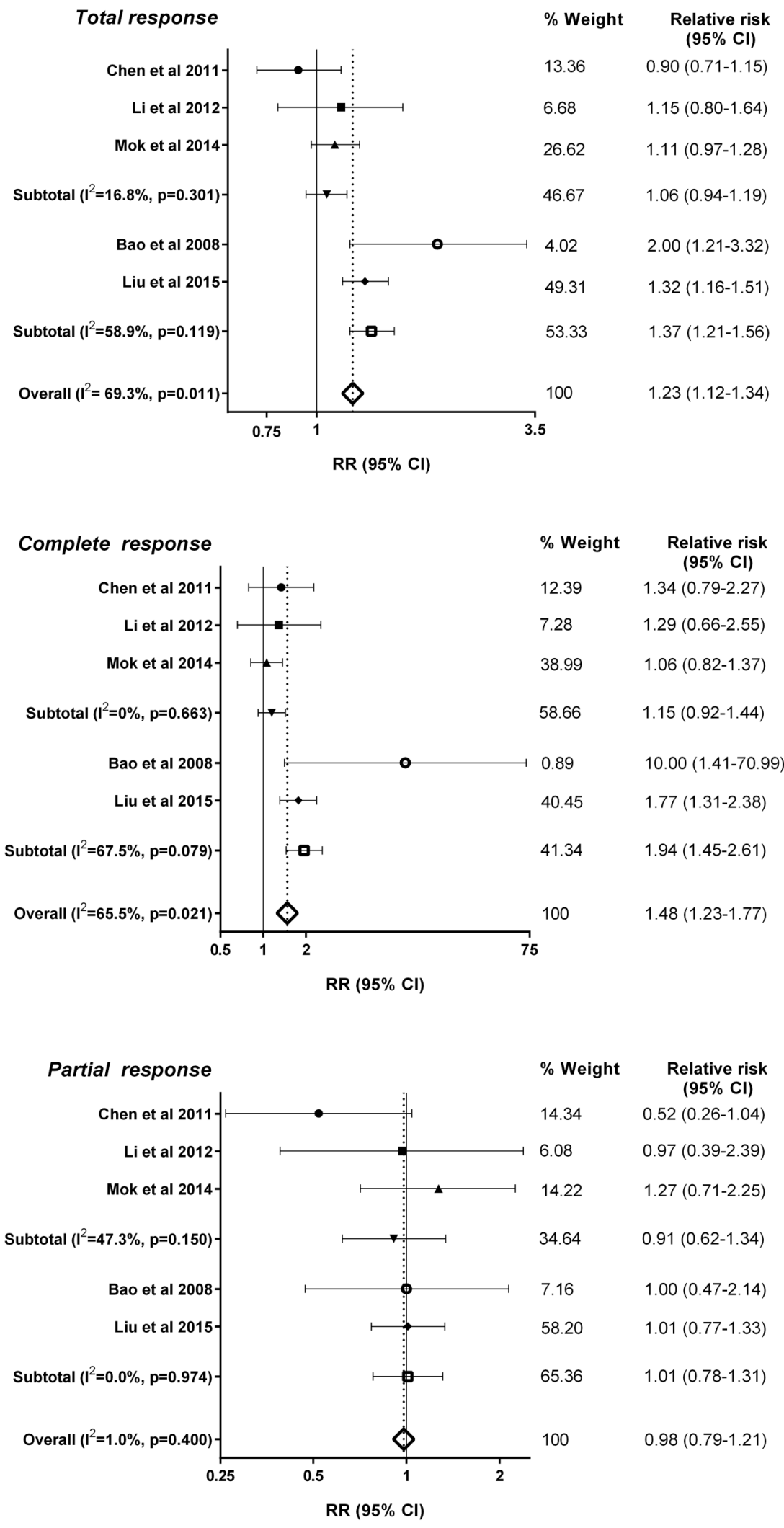

Figure 2 Forest plots of the relative risks (RRs) and 95\% Cls of the total (complete plus partial), complete and partial renal response rates in the selected randomised controlled trials (RCTs) upon induction tacrolimus-based treatment versus conventional treatment. A fixed-effects meta-analysis was performed. The meta-analysis was performed for studies using duo therapy (adapted from Mok et al [8], Chen et al [18], Li et al [19]) and for studies using triple therapy (adapted from Liu et al [9], Bao et al [20]) separately as well. The vertical solid line represents an RR of 1 and the dotted line illustrates the overall RR. The $p$ value of the test for heterogeneity is shown for subtotal and overall analyses. 

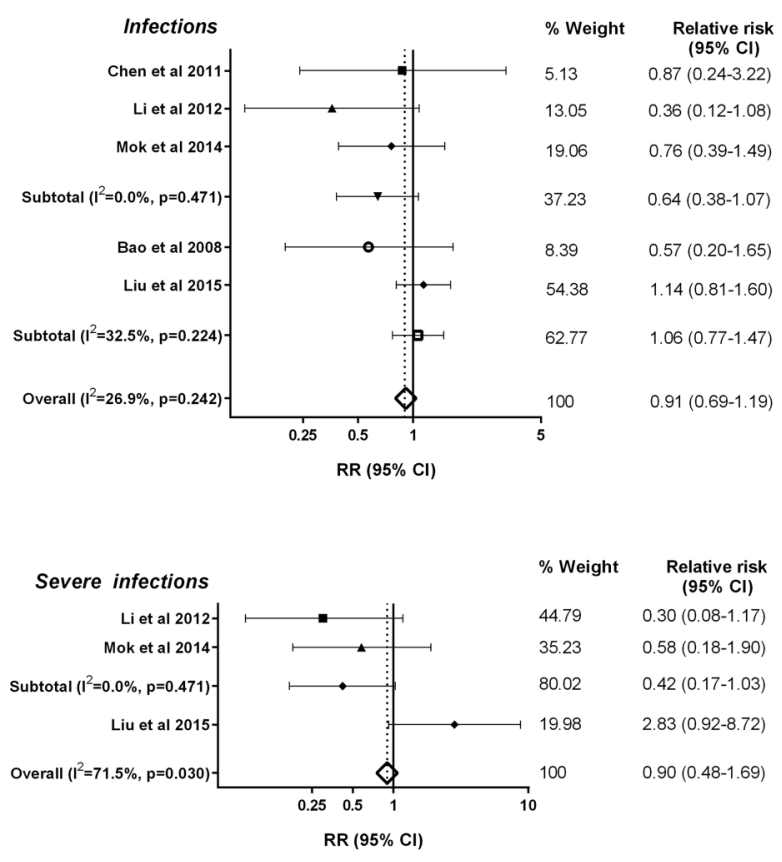
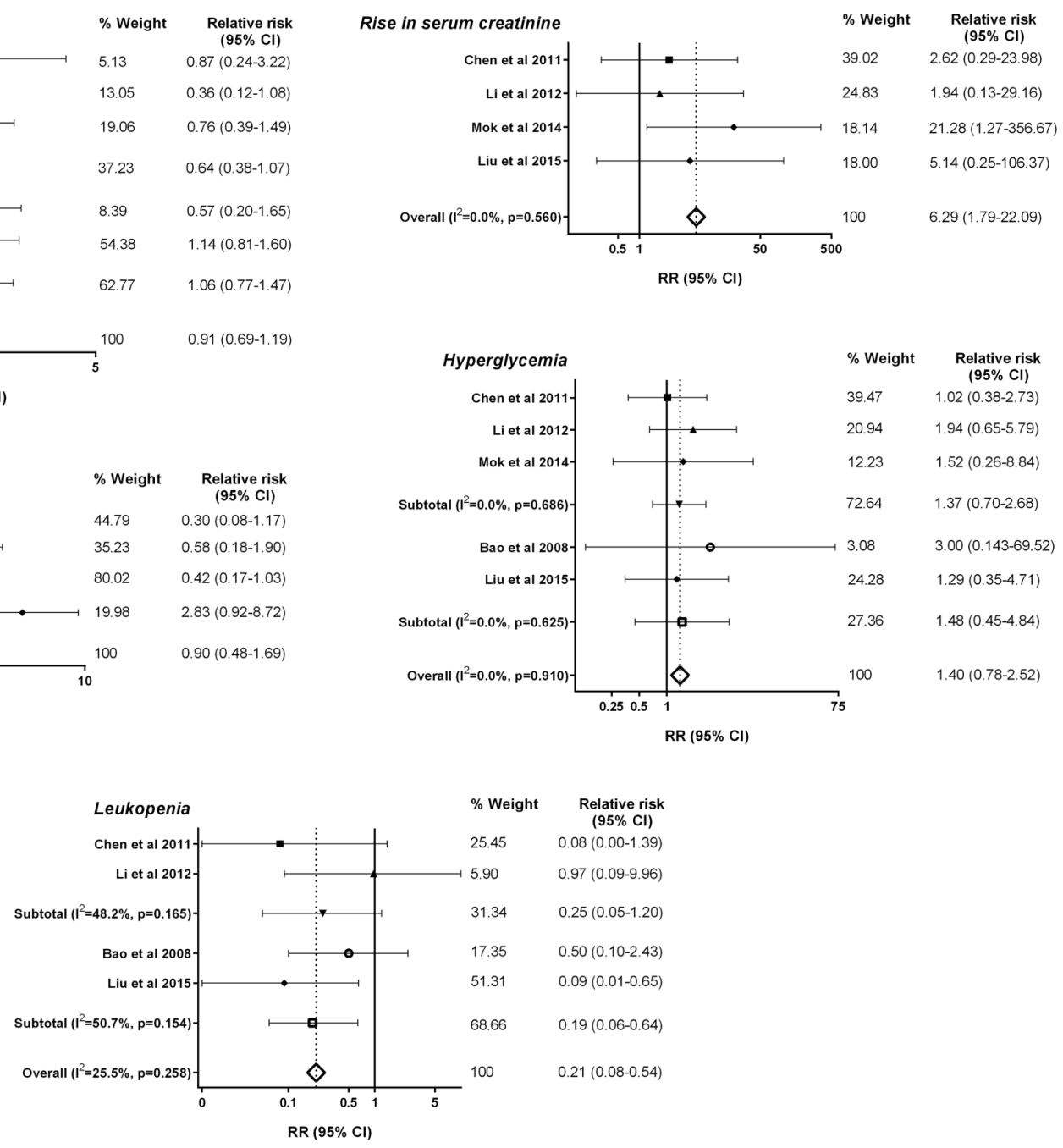

Figure 3 Forest plots of the relative risks (RRs) and 95\% Cls for the five most commonly reported adverse events in the selected randomised controlled trials (RCTs) on tacrolimus-based treatment versus conventional treatment. Overall infections, severe infections, hyperglycaemia, leucopoenia and rise in serum creatine were used in a meta-analysis, using a fixed-effects model. For infections, hyperglycaemia and leucopoenia, a meta-analysis was performed for studies using duo therapy (adapted from Mok et al [8], Chen et al [18], Li et al [19]) and for studies using triple therapy (adapted from Liu et al [9], Bao et al [20]) separately as well. The vertical solid line represents an RR of 1 and the dotted line illustrates the overall RR. The $p$ value of the test for heterogeneity is shown for subtotal and overall analyses.

CNIs were studied in LN before. Early exploratory studies on the efficacy of ciclosporine in LN resulted in comparable efficacy to conventional treatments, at the cost of unacceptably higher adverse events rates. ${ }^{38}$ However, a small RCT in 40 patients (Cyclofa-Lune trial ${ }^{39}$ demonstrated that after approximately 8 years of follow-up, ciclosporine was non-inferior to high-dose cyclophosphamide as induction treatment for proliferative LN. A second $\mathrm{RCT}^{40}$ in class $\mathrm{V}$ membranous $\mathrm{LN}$, showed faster remission with ciclosporine compared with cyclophosphamide with comparable remission rates. Long-term follow-up of 5 years showed increased relapse rates in the ciclosporine treated arm. Only one study that investigated ciclosporine as maintenance therapy observed equal efficacy to azathioprine in preventing disease flares. ${ }^{41}$ On a histopathological level, ciclosporine was unable to reduce chronic activity in lupus kidney biopsies, supporting the hypothesis that the antiproteinuric effects of ciclosporine were predominantly attributable to haemodynamic rather than immunological changes. ${ }^{42}$ We know from the vast literature on transplantation that ciclosporine and TAC are different with respect to immunological efficacy as well as safety profile. Ciclosporine binds cyclophilin while TAC binds FK506, resulting in different immunosuppressive effects. ${ }^{43}$ Furthermore, both ciclosporine and TAC have small therapeutic widths, causing small variations in dosing to potentially imply large differences in efficacy and toxicity. Therefore, irrespective of the available data on ciclosporine, further investigations into the efficacy of TAC on clinical as well as histopathological end points are clearly warranted.

There are important limitations to consider in the present meta-analysis. First, the quality of the controlled 
studies was low as defined by the Delphi score, mainly because of the incomplete blinding procedures in all studies. Second, as mentioned before, from five RCTs the largest RCT performed by Liu et at determined $49 \%$ of the overall total response. Third, TAC regimens were heterogeneous across all studies: target trough levels varied or were not used and also concomitant steroid dosing differed (see online supplementary table S2). This notion hampers a general recommendation on the optimal dosing of TAC. Fourth, no long-term results could be investigated in this meta-analysis. Only one study ${ }^{8}$ reported long-term results (ie, 5 years of follow-up) after induction treatment with TAC and prednisone during 6 months followed by azathioprine and prednisone as maintenance treatment. Of note, a higher rate of renal relapses was observed in the TAC-based treatment arm, which did not reach statistical significance $(p=0.13)$. Lastly, it needs to be emphasised that all included studies were performed in Asian patients. The importance of ethnicity has been demonstrated by the ethnicity-based subgroup analysis of the Aspreva Lupus Management Study (ALMS) trial. ${ }^{44}$ Superiority of mycophenolate over cyclophosphamide was predominantly determined by its efficacy in African-American and Hispanic patients. In addition, genome-wide association studies revealed different genetic susceptibility loci for SLE between ethnicity groups. ${ }^{45}$ Also, the CYP3A5 polymorphism determines the metabolism of TAC, and a lower bioavailability of TAC in African-American kidney transplant recipients ${ }^{46} 47$ is well described. Altogether emphasising that the extrapolation of these data to other ethnic groups is not selfevident. Of note, we found only three non-controlled case series treating non-Asian subjects with TAC-based regimens. ${ }^{24-26}$ Despite these limitations, this comprehensive analysis of all published studies illustrated that TAC-based therapy in selected patients with LN can be efficacious without major safety concerns. Therefore, these data emphasise the importance to further investigate the efficacy of TAC for patients with active LN.

Indeed, the international Lupus Trial Nephritis Network has recently initiated the design of a trial with a TAC-based regimen. In this respect a few considerations could be deducted from our current study. Based on the efficacy results in our meta-analysis, it would be plausible to investigate triple therapy randomising a multiethnic patient population with LN. Regarding safety of such an RCT, a possible higher risk for severe infections in the triple therapy arm needs to be monitored closely. The general dosing in a TAC regimen is roughly estimated at 3-4 mg twice daily during the induction phase, based on the summary of studies. Monitoring of trough levels is not mandatory although it can help to exclude low exposition in patients. Most importantly, the definition of the primary renal end point needs much attention: due to the haemodynamic effects of TAC on reducing proteinuria, as discussed above, the classic LN renal end point which is mainly based on proteinuria improvement is intrinsically biased. Briefly, patients with LN with TAC have a quick reduction of proteinuria within the first weeks of treatment (most probably due to haemodynamic effects) and therefore empirically a higher chance of achieving a partial or even complete response. Also in the current meta-analysis, we could not exclude whether a haemodynamic effect is (partly) responsible for the positive effects of TAC regimens. While a haemodynamic effect is undoubtedly present, it seems unlikely that this effect could fully explain the beneficial effect of TAC-based therapies. Thus, it seems wise to consider a less biased renal end point such as a repeat renal biopsy. Although timing of this renal biopsy needs careful consideration, it would confirm treatment efficacy in a more objective manner plus help to identify whether, if any, there is a risk for CNI toxicity on the renal tissue level. Of note, it is important to take into account that all included studies in this study used proteinuria as an important remission criterion (see online supplementary table S2). Taking these considerations into account, a multicentre, international RCT defining the role of TAC in LN treatment should be feasible and eagerly embraced by the scientific community.

In conclusion, we recommend the use of a TAC-based regimen in the selected group of Asian patients with LN. In addition, we recommend considering a TAC-based regimen in the subgroups of patients with refractory LN and (pre)pregnant patients with LN. Although long-term efficacy and safety results are lacking, it seems reasonable to conclude that when patients with LN are to be treated with TAC, this strategy seems not to be inferior to conventional treatment and has a good safety profile. In the future, the place of TAC in the therapeutic armamentarium for LN can only be established when a multicentre, international RCT is performed as now proposed by the international Lupus Nephritis Trial Network.

\section{Author affiliations}

${ }^{1}$ Department of Nephrology, Leiden University Medical Center, Leiden, The Netherlands

${ }^{2}$ Clinic for Lupus-, Vasculitis and Complement-Mediated Systemic Diseases, Leiden University Medical Center, Leiden, The Netherlands

${ }^{3}$ Department of Gerontology and Geriatrics, Leiden University Medical Center, Leiden, The Netherlands

${ }^{4}$ Department of Cardiology, Leiden University Medical Center, Leiden, The Netherlands

${ }^{5}$ Department of Rheumatology, Leiden University Medical Center, Leiden, The Netherlands

Acknowledgements The authors thank J Schoones for his advice and support with the literature search. The authors also thank Professor Dr O M Dekkers for independently reviewing statistical analysis.

Contributors TK, TJR, YKOT contributed to the ideas for the article. YKOT performed the literature search. TK, AJMC, ST and YKOT contributed to data acquisition and analysis. TK, OWB, ST, TWJH, TJR and YKOT contributed to the writing of the manuscript. YKOT is the guarantor.

Funding The work of TK and YKOT was supported by the Dutch Kidney Foundation (KJPB12.028) and a Clinical Fellowship by the Dutch Organisation for Scientific Research.

Competing interests None declared. 
Provenance and peer review Not commissioned; externally peer reviewed.

Data sharing statement No additional data are available.

Open Access This is an Open Access article distributed in accordance with the Creative Commons Attribution Non Commercial (CC BY-NC 4.0) license, which permits others to distribute, remix, adapt, build upon this work noncommercially, and license their derivative works on different terms, provided the original work is properly cited and the use is non-commercial. See: http:// creativecommons.org/licenses/by-nc/4.0/

\section{REFERENCES}

1. Seligman VA, Lum RF, Olson JL, et al. Demographic differences in the development of lupus nephritis: A retrospective analysis. Am J Med 2002;112:726-9.

2. Bernatsky S, Boivin JF, Joseph L, et al. Mortality in systemic lupus erythematosus. Arthritis Rheum 2006;54:2550-7.

3. Hahn BH, McMahon MA, Wilkinson A, et al., American College of Rheumatology. American College of Rheumatology guidelines for screening, treatment, and management of lupus nephritis. Arthritis Care Res (Hoboken) 2012;64:797-808.

4. Bertsias GK, Tektonidou M, Amoura Z, et al. Joint European League Against Rheumatism and European Renal Association-European Dialysis and Transplant Association (EULAR/ERA-EDTA) recommendations for the management of adult and paediatric lupus nephritis. Ann Rheum Dis 2012;71:1771-82.

5. Tamirou F, D'Cruz D, Sangle S, et al. Long-term follow-up of the MAINTAIN Nephritis Trial, comparing azathioprine and mycophenolate mofetil as maintenance therapy of lupus nephritis. Ann Rheum Dis 2016;75:526-31.

6. Rovin $\mathrm{BH}$, Furie R, Latinis $\mathrm{K}$, et al. Efficacy and safety of rituximab in patients with active proliferative lupus nephritis: the Lupus Nephritis Assessment with Rituximab study. Arthritis Rheum 2012;64:1215-26.

7. Merrill JT, Neuwelt CM, Wallace DJ, et al. Efficacy and safety of rituximab in moderately-to-severely active systemic lupus erythematosus: the randomized, double-blind, phase II/III systemic lupus erythematosus evaluation of rituximab trial. Arthritis Rheum 2010;62:222-33.

8. Mok CC, Ying KY, Yim CW, et al. Tacrolimus versus mycophenolate mofetil for induction therapy of lupus nephritis: a randomised controlled trial and long-term follow-up. Ann Rheum Dis 2016;75:30-6.

9. Liu Z, Zhang H, Liu Z, et al. Multitarget Therapy for Induction Treatment of Lupus Nephritis. Ann Intern Med 2015;162:18-26.

10. EBPG Expert Group on Renal Transplantation. European best practice guidelines for renal transplantation. Section IV: Long-term management of the transplant recipient. IV.10. Pregnancy in renal transplant recipients. Nephrol Dial Transplant 2002;17(Suppl 4):50-5.

11. Kidney Disease: Improving Global Outcomes (KDIGO) Transplant Work Group. KDIGO clinical practice guideline for the care of kidney transplant recipients. Am J Transplant 2009;9(Suppl 3) S1-155.

12. Mendez R. FK 506 and mycophenolate mofetil in renal transplant recipients: six-month results of a multicenter, randomized dose ranging trial. FK 506 MMF Dose-Ranging Kidney Transplant Study Group. Transplant Proc 1998;30:1287-9.

13. Scott LJ, McKeage K, Keam SJ, et al. Tacrolimus: a further update of its use in the management of organ transplantation. Drugs 2003;63:1247-97.

14. Naesens M, Kuypers DRJ, Sarwal M. Calcineurin inhibitor nephrotoxicity. Clin J Am Soc Nephrol 2009;4:481-508.

15. Entani $\mathrm{C}$, Izumino $\mathrm{K}$, lida $\mathrm{H}$, et al. Effect of a novel immunosuppressant, FK506, on spontaneous lupus nephritis in MRL/MpJ-Ipr/Ipr mice. Nephron 1993;64:471-5.

16. Moher D, Liberati A, Tetzlaff J, et al. Preferred Reporting Items for Systematic Reviews and Meta-Analyses: the Preferred Reporting Items for Systematic Reviews and Meta-Analyses (PRISMA) statement. Ann Intern Med 2014;151:264-9.

17. Verhagen AP, De Vet HCW, De Bie RA, et al. The Delphi list: a criteria list for quality assessment of randomized clinical trials for conducting systematic reviews developed by Delphi consensus. J Clin Epidemiol 1998;51:1235-41.

18. Chen W, Tang X, Liu Q, et al. Short-term outcomes of induction therapy with tacrolimus versus cyclophosphamide for active lupus nephritis: A multicenter randomized clinical trial. Am J Kidney Dis 2011;57:235-44.
19. Li X, Ren H, Zhang Q, et al. Mycophenolate mofetil or tacrolimus compared with intravenous cyclophosphamide in the induction treatment for active lupus nephritis. Nephrol Dial Transplant 2012;27:1467-72.

20. Bao H, Liu ZH, Xie HL, et al. Successful treatment of class $\mathrm{V}+\mathrm{IV}$ lupus nephritis with multitarget therapy. J Am Soc Nephrol 2008;19:2001-10.

21. Chen W, Liu Q, Chen W, et al. Outcomes of maintenance therapy with tacrolimus versus azathioprine for active lupus nephritis: a multicenter randomized clinical trial. Lupus 2012;21:944-52.

22. Yap DY, Yu X, Chen XM, et al. Pilot 24 month study to compare mycophenolate mofetil and tacrolimus in the treatment of membranous lupus nephritis with nephrotic syndrome. Nephrology (Carlton) 2012;17:352-7.

23. Wang S, Li X, Qu L, et al. Tacrolimus versus cyclophosphamide as treatment for diffuse proliferative or membranous lupus nephritis: a non-randomized prospective cohort study. Lupus 2012;21:1025-35.

24. Lanata CM, Mahmood T, Fine DM, et al. Combination therapy of mycophenolate mofetil and tacrolimus in lupus nephritis. Lupus 2010;19:935-40.

25. Gordon S, Denunzio T, Uy A. Success using tacrolimus in patients with proliferative and membranous lupus nephritis and refractory proteinuria. Hawaii J Med Public Health 2013;72:18-23. http://www. pubmedcentral.nih.gov/articlerender.fcgi? artid $=3764582 \&$ tool=pmcentrez\& rendertype $=$ abstract

26. Cortés-Hernández J, Torres-Salido MT, Medrano AS, et al. Long-term outcomes-Mycophenolate mofetil treatment for lupus nephritis with addition of tacrolimus for resistant cases. Nephrol Dial Transplant 2010;25:3939-48.

27. Lee YH, Song GG. Relative efficacy and safety of tacrolimus, mycophenolate mofetil, and cyclophosphamide as induction therapy for lupus nephritis: a Bayesian network meta-analysis of randomized controlled trials. Lupus 2015;24:1520-8.

28. Hannah J, Casian A, D'Cruz D. Tacrolimus use in lupus nephritis: a systematic review and meta-analysis. Autoimmun Rev 2016;15:93-101.

29. Shekelle $\mathrm{P}$, Woolf $\mathrm{S}$, Eccles $\mathrm{M}$, et al. Clinical guidelines: developing guidelines. BMJ 1999;318:593-6. http://www.ncbi.nlm.nih.gov/pmc/ articles/PMC1115034/pdf/593.pdf

30. van Tellingen A, Voskuyl AE, Vervloet MG, et al., Dutch Working Party on Systemic Lupus Erythematosus. Dutch guidelines for diagnosis and therapy of proliferative lupus nephritis. Neth $J$ Med 2012;70:199-206.

31. Tunnicliffe DJ, Singh-Grewal D, Kim S, et al. Diagnosis, monitoring and treatment of systemic lupus erythematosus: a systematic review of clinical practice guidelines. Arthritis Care Res (Hoboken) 2015;67:1440-52

32. Singh JA, Hossain A, Kotb A, et al. Risk of serious infections with immunosuppressive drugs and glucocorticoids for lupus nephritis : a systematic review and network meta-analysis. BMC Med 2016;14:137.

33. Webster $\mathrm{P}$, Wardle A, Bramham $\mathrm{K}$, et al. Tacrolimus is an effective treatment for lupus nephritis in pregnancy. Lupus 2014;23: 1192-6.

34. Christopher V, Al-Chalabi T, Richardson PD, et al. Pregnancy outcome after liver transplantation: a single-center experience of 71 pregnancies in 45 recipients. Liver Transplant 2006;12:1138-43.

35. Kainz A, Harabacz I, Cowlrick IS, et al. Review of the course and outcome of 100 pregnancies in 84 women treated with tacrolimus. Transplantation 2000;70:1718-21.

36. Bramham K, Soh MC, Nelson-Piercy C. Pregnancy and renal outcomes in lupus nephritis: an update and guide to management. Lupus 2012;21:1271-83.

37. Grootscholten C, Ligtenberg G, Hagen EC, et al. Azathioprine/ methylprednisolone versus cyclophosphamide in proliferative lupus nephritis. A randomized controlled trial. Kidney Int 2006;70: 732-42.

38. Isenberg DA, Snaith ML, Morrow WJ, et al. Cyclosporin A for the treatment of systemic lupus erythematosus. Int $J$ Immunopharmacol 1981;3:163-9.

39. Zavada J, Pesickova S, Rysava R, et al. Cyclosporine A or intravenous cyclophosphamide for lupus nephritis: the Cyclofa-Lune study. Lupus 2010;19:1281-9.

40. Austin HA, Illei GG, Braun MJ, et al. Randomized, controlled trial of prednisone, cyclophosphamide, and cyclosporine in lupus membranous nephropathy. J Am Soc Nephrol 2009;20:901-11.

41. Moroni G, Doria A, Mosca M, et al. A randomized pilot trial comparing cyclosporine and azathioprine for maintenance therapy in diffuse lupus nephritis over four years. Clin J Am Soc Nephrol 2006;1:925-32. 
42. Radhakrishnan J, Kunis CL, D'Agati V, et al. Cyclosporine treatment of lupus membranous nephropathy. Clin Nephrol 1994;42:147-54. http://www.ncbi.nlm.nih.gov/entrez/query.fcgi?cmd=Retrieve\&db= PubMed\&dopt=Citation\&list uids $=7994932$

43. Almawi WY, Melemedjian OK. Clinical and mechanistic differences between FK506 (tacrolimus) and cyclosporin A. Nephrol Dial Transplant 2000;15:1916-8.

44. Appel GB, Contreras G, Dooley MA, et al. Mycophenolate mofetil versus cyclophosphamide for induction treatment of lupus nephritis. J Am Soc Nephrol 2009;20:1103-12.
45. Sánchez E, Comeau ME, Freedman BI, et al. Identification of novel genetic susceptibility loci in African American lupus patients in a candidate gene association study. Arthritis Rheum 2011;63:3493-501.

46. Dirks NL, Huth B, Yates CR, et al. Pharmacokinetics of immunosuppressants: a perspective on ethnic differences. Int J Clin Pharmacol Ther 2004;42:701-18.

47. Provenzani A, Santeusanio A, Mathis E, et al. Pharmacogenetic considerations for optimizing tacrolimus dosing in liver and kidney transplant patients. World J Gastroenterol 2013;19:9156-73. 